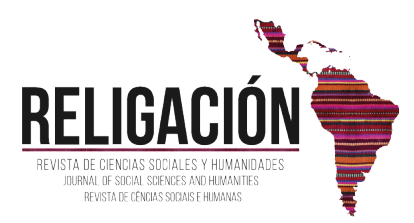

Sección General

\title{
La Formación Docente Socioformativa para el Desarrollo Social Sostenible
}

\author{
Socioformative Teacher Training for Sustainable Social Development
}

RESUMEN: El presente artículo constituye un análisis sobre los retos que representan las sociedades del siglo XXI, Aborda aspectos claves para orientar el aprovechamiento de los nuevos enfoques sistémicos, destacando el enfoque socioformativo, y que son debate de diversas investigaciones a nivel internacional. El argumento utilizado, permite identificar el debate académico respecto a dicho enfoque y como sus características están orientadas a la construcción y desarrollo de sociedades sostenibles; de la misma manera, permite verificar las relaciones en escenarios, contextos reales, donde es necesario mostrar una capacidad autentica para afrontar los retos y peligros de nuestro entorno. Contempla una visión educativa desde tres directrices: el currículo escolar, formación docente y formación continua. El sistema educativo, es aliado para erigir la transformación hacia un enfoque socioformativo eficaz, que contribuya a generar una sociedad más humana, capaz de atender los problemas reales que nos aquejan ahora y en el futuro.

Palabras clave: desarrollo sostenible, formación docente, formación continua, sociedad del conocimiento, socioformación

ABSTRACT: This article constitutes an analysis of the challenges that the societies of the 21 st century represent. It addresses key aspects to guide the use of new systemic approaches, highlighting the socioformative approach, and which are the debates of various investigations at an international level. The argument used allows us to identify the academic debate regarding said approach and how its characteristics are oriented to the construction and development of sustainable societies; in the same way, it allows to verify relationships in scenarios, real contexts, where it is necessary to show an authentic capacity to face the challenges and dangers of our environment. It contemplates an educational vision from three guidelines: the school curriculum, teacher training and continuous training. The educational system is an ally to erect the transformation towards an effective socioformative approach, which contributes to generate a more human society, capable of addressing the real problems that afflict us now and in the future.

Keywords: sustainable development, teacher training, lifelong learning, knowledge society, socioformation 


\section{INTRODUCCIÓN}

El presente artículo tiene como finalidad, realizar un análisis documental sobre el concepto y enfoque actual del paradigma socioformativo y el desarrollo sostenible. El tema propuesto, conjuga la perspectiva que se tiene respecto a estos enfoques, desde la formación docente, el currículo educativo y la formación continua; y cómo a partir de estas directrices se establecen sus relaciones y su vínculo con el desarrollo sostenible, por lo que es necesario clarificar los conceptos de sociedad del conocimiento, socioformación y desarrollo sostenible.

Para ello es preciso revisar en nuestra investigación, cómo se conceptualizan las sociedades de la información y del conocimiento, así como el papel que han desempeñado históricamente; también es necesario valorar los paradigmas educativos que se mantienen en el currículo escolar y su reflejo en las prácticas docentes, el lazo establecido por la misma formación docente, y la profesionalización a través de programas de formación continua, verificado si estos contribuyen con un desarrollo sostenible, posibilitando a los actores del sistema educativo para enfrentar retos cada vez más complejos.

Partimos por entender las concepciones actuales sobre las sociedades de la información y del conocimiento, lo cual implica transitar en un contexto definido a finales del siglo XX y en el inicio del siglo XXI, donde se provee un notorio avance de la ciencia y la tecnología (Mañas, 2019). esto nos constituye para ubicarnos dentro de una sociedad de información, configurada por la producción y circulación de un incesante flujo de datos. Involucra entonces, la necesidad de reconocer las características de dicha sociedad y transitar de dicha sociedad de la información hacia una sociedad del conocimiento (Olivé \& Ransanz, 2011). Dicha transición a este tipo de sociedad, se ve representada por la generación de una conciencia humana, una visión más amplia, capaz de resolver problemas de su entorno para fortalecer un desarrollo sostenible (Rendón, Escobar, Arango, Molina, Villamil y Valencia, 2018); Es necesario entonces, aprovechar los espacios escolares a través del trabajo docente para fortalecer esta perspectiva. (UNESCO, 2005).

Para orientar los retos derivados entre la sociedad de la información y sociedad del conocimiento, es necesario clarificar el concepto de socioformación y el enfoque actual sobre el mismo considerando su etimología, "Social" del lat. sociĕtas, -ātis agrupación natural o pactada de personas, organizada para cooperar en la consecución de determinados fines, y "formar" del lat. formāre. dicho de una persona, adquirir preparación intelectual, moral o profesional (RAE, 2018). Bajo esta premisa, el nuevo enfoque considera que las personas sean capaces de poner en juego competencias más amplias (intelectuales, morales o profesionales), que les permita resolver problemas de su contexto real, de su entorno inmediato; los cuales, muchas veces se encuentra ajenos al contexto en el que estos se plantean en el ámbito escolar (Tobón, González, Nambo, \& Antonio, 2015).

Lo señalado anteriormente nos remite a clarificar el concepto de contexto. El término "contexto", del lat. contextus, se refiere al entorno físico o de situación, político, histórico, cultural o de cualquier otra índole, en el que se considera un hecho (RAE, 2019). Es por esto que, al referirnos a un problema, no se trata de verlo o enfrentarlo, desde una visión simple; o más aún, de enfrentarlo haciendo uso de conceptos estrictamente de carácter cognoscitivo. El contexto pone en acción a un sujeto con su entorno, dicho entorno es real, y se plantea desde diferentes tramas de interacción humana, la familia, la comunidad, la sociedad, lo ambiental y ecológico, etc. Es decir, un espacio adecuado que somete a los estudiantes o personas, a movilizar saberes de manera adecuada y pertinente, para encontrar soluciones a dichos problemas. (Tobón, 2010).

Los problemas actuales del contexto, evolucionan conforme existen avances en el desarrollo de la ciencia y la tecnología, se trata de problemas abiertos, complejos y controvertidos (España \& Prieto, 2009), los cuales requieren de una multiplicidad de elementos, ideas o conceptos, sin que estos contribuyan en la obtención de una idea específica cualquiera que sea la solución dada o posturas que se fijen sobre estos, siempre estarán sujetos a un debate o nuevas argumentaciones; estos se incrementaran con el desarrollo científico y tecnológico. (Tobón, 2010). Por tal razón nos planteamos algunos cuestionamientos; ¿se tendrá clara la visión de los problemas reales del contexto desde una perspectiva social?, ¿será posible enfrentar estos retos desde los enfoques socioeducativos actuales?, ¿contribuirá la educación que se brinda en México, afrontar estos retos?

Al plantearnos estas interrogantes, es necesario valorar los problemas reales que nos aquejan hoy día, aquellos problemas que representan retos, desde diversos puntos de vista (económicos, políticos, sociales, ambientales, de salud, etc.), y que ponen en peligro la continuidad de la existencia humana. Por ello, es necesario hacer un alto en el tema del capitalismo histórico, pues es aquí donde nace la proliferación de una sociedad consumista (Wallerstein, 1988), incapaz de reflexionar sobre los riegos que nos aquejan; y que, actualmente, mantiene el uso indiscriminado de los recursos naturales en diversas latitudes del planeta, generando así su mayor impacto en el deterioro ambiental, pues existen estudios referentes al problema del Cambio Climático y la disminución crítica de recursos como el agua potable, lo cual deja al descubierto la generación de nuevos problemas y desafíos cada vez más complejos de atender (Luna-Nemecio, 2019)

La educación ha sido, es y será el motor de transformación en los países para su desarrollo, en México, el currículo educativo actual, considera un enfoque competencial sostenido en cuatro pilares de la educación, "apren- 
der a conocer", "aprender a hacer", "aprender a ser" y "aprender a vivir juntos" (Delors, 1993). Dicho enfoque fue implementado en la educación básica desde el 2004 en el nivel preescolar, y de manera progresiva en los demás niveles educativos. A finales de la década de los noventas ya era patente la implementación del enfoque competencial en el nivel medio superior; para ahondar en la solución de este problema, es necesario realizar un análisis más profundo de manera retrospectiva sobre los modelos educativos establecidos a mediados del siglo XIX, en los cuales, sus perspectivas estaban orientadas a la atención de la época con las sociedades industriales. (Hernández, Tobón, \& Vazquez, 2014)

A partir del desarrollo científico y tecnológico, en la década de los setentas se incorpora a los nuevos paradigmas, los retos que contenían el desarrollo de las nacientes sociedades de la información. De esta forma, podemos entender por qué en la mayoría de las instituciones escolares, prevalece la enseñanza puntual de contenidos; no obstante, los planes de estudio actualmente, establecen principios pedagógicos para abordar temas de relevancia social, como son: educación ambiental, educación financiera, educación para la salud, entre otros (SEP, 2011, pág. 36) ; sin embargo, en la práctica docente se reduce a la exposición de contenidos como unidades de análisis, sin observar los retos implícitos para un desarrollo sostenible. (Hernández, et al 2014)

Más aún, esta visión prevalece en la mayoría de los sistemas educativos latinoamericanos formadores de docentes, un ejemplo de ello, lo podemos apreciar en la malla curricular definida para docentes de educación básica en México (DGESPE, 2018), al hacer su revisión y análisis, podemos apreciar que falta incluir áreas donde se desarrolle el enfoque socioformativo, necesario en la formación docente; pues desde este mismo, se contribuiría en la atención que demandan cada vez más los retos que implican las sociedades del conocimiento, en una extensión globalizada, "en este contexto, la sociedad del conocimiento se combina con la globalización. La globalización es un proceso que comprende múltiples dimensiones, tales como los aspectos económicos, sociales, políticos y culturales." (Pedraja Rejas, 2012).

Una oportunidad para brindar atención a este vacío en la formación docente, es el sistema de formación continua, el cual brinda elementos para la mejora profesional y la posibilidad de incorporar nuevos conocimientos y experiencias a la práctica docente; no obstante, sus temáticas siempre se han orientado a la atención de aspectos disciplinares, al abordaje de contenidos, o diseño de estrategias didácticas, y aunque se han realizados acciones para que los docentes a través de un análisis breve, reconozcan sus propias necesidades de actualización o profesionalización; continua sin imperar una noción clara para desarrollar un enfoque apegado a las sociedad del conocimiento.

Las comunidades de aprendizaje, son un mecanismo de interacción probado como respuesta a los cambios y retos sociales que han sido acuñados por la sociedad de la información (Díez-Palomar \& Flecha García, 2010). La apertura a espacios de interacción, diálogo y comunidad, permite prepararse en la competitividad y la solidaridad, aspectos fundamentales para enfrentar los retos del contexto actual, pero sin perder de vista el enfoque que permita un desarrollo sostenible, procurando así convertirse en una sociedad del conocimiento eficaz, pero sobre todo valorando los riesgos de las decisiones humanas que nos afectan como sociedad, este concepto incluye al trabajo no solo de profesionales de una misma rama, sino de la integración de personas adultas, pues existe en ellos el bagaje de conocimientos adquiridos por la inteligencia cultural. (Racionero \& Serradell, 2005)

\section{OBJETIVOS}

A partir de los diversos referentes teóricos e investigaciones científicas analizadas, proponemos como metas de esta investigación las siguientes:

1. Exponer las concepciones que identifican las características de la formación docente socioformativa y el desarrollo sostenible.

2. Determinar la relación del desarrollo sostenible y su vinculación en la sociedad del conocimiento, desde la formación docente

3. Proponer como estrategias a las comunidades de aprendizaje para que, desde la formación continua, fomentar el diálogo que permita situarnos en una sociedad del conocimiento y afrontar retos para el desarrollo sostenible.

\section{METODOLOGÍA}

El estudio realizado se hizo mediante un análisis documental sobre formación docente desde el enfoque socioformativo. El análisis documental permite la sistematización unificada de los documentos (Dulzaides \& Molina, 
2019), mismo que sirvieron de apoyo a esta investigación y fueron recuperados de buscadores académicos, libros, revistas y publicaciones electrónicas.

Para efectuar la búsqueda de información respecto a nuestra investigación, se establecieron cuatro categorías de análisis como ejes centrales del tema, este permitió construir una estrategia para sistematizar, organizar, construir y comunicar los hallazgos relevantes. Por lo extenso de las categorías investigadas, fue necesario establecer subcategorías refiriendo componentes para su análisis, dicha información fue organizada en una tabla para su correcto seguimiento. (ver Tabla 1).

TABLA 1. Análisis de Categorías Empleadas en el Estudio

\begin{tabular}{|c|c|c|}
\hline CATEGORÍAS & SUBCATEGORÍAS & PREGUNTAS O COMPONENTES \\
\hline Socioformación & $\begin{array}{l}\text {-Concepto de socioformación. } \\
\text {-Historicidad de socioformación. } \\
\text {-Concepto de sociedad del conocimiento. } \\
\text {-Vinculación de la socioformación y la } \\
\text { sociedad del conocimiento. }\end{array}$ & $\begin{array}{l}\text {-Etimología de la palabra socioformación. } \\
\text {-Desarrollo histórico. } \\
\text {-Definición actual de socioformación. } \\
\text {-ldentificación de las características clave que definen una } \\
\text { sociedad del conocimiento. } \\
\text {-Explicación de cada una de las características } \\
\text { encontradas. } \\
\text {-Relación de las sociedades del conocimiento con el } \\
\text { enfoque socioformativo. }\end{array}$ \\
\hline Formación docente & $\begin{array}{l}\text {-Concepciones de formación docente. } \\
\text {-Competencias docentes y su vínculo con } \\
\text { la sociedad del conocimiento. } \\
\text {-Desafíos para el docente en la sociedad } \\
\text { del conocimiento. }\end{array}$ & $\begin{array}{l}\text {-Definición de formación docente } \\
\text { desarrollo sostenible. } \\
\text {-Desarrollo histórico. } \\
\text {-Definición actual. } \\
\text {-Relación de la formación docente y el enfoque } \\
\text { socioformativo. } \\
\text {-Críticas al modelo actual de formación, capacitación y } \\
\text { profesionalización. } \\
\text {-Retos de la educación del siglo XXI para la sostenibilidad. }\end{array}$ \\
\hline Desarrollo sostenible & $\begin{array}{l}\text {-Concepto de desarrollo sostenible. } \\
\text {-Historicidad del desarrollo sostenible. } \\
\text {-Complejidad social y el desarrollo } \\
\text { sostenible. }\end{array}$ & $\begin{array}{l}\text {-Concepto de desarrollo sostenible. } \\
\text {-Desarrollo histórico. } \\
\text {-Definición actual. } \\
\text {-Retos de las sociedades actuales para la sostenibilidad. }\end{array}$ \\
\hline $\begin{array}{l}\text { Comunidades de } \\
\text { aprendizaje }\end{array}$ & $\begin{array}{l}\text {-Concepto de comunidad de aprendizaje } \\
\text {-Espacios dialógico }\end{array}$ & $\begin{array}{l}\text {-Definición de comunidad de aprendizaje. } \\
\text {-Desarrollo histórico. } \\
\text {-Definición actual. } \\
\text {-Dialogicidad. } \\
\text {-Problemas complejos. }\end{array}$ \\
\hline
\end{tabular}

Elaborado por Dany Vázquez-Ayala para la presente investigación

Se realizó un búsqueda y análisis exhaustivo de artículos y libros apoyándonos en bases de datos como fueron: Google Académico, Scielo, Redalyc, entre otras; para su búsqueda fue necesario emplear palabras esenciales como, "socioformación", "enfoque socioformativo", "retos de las sociedades actuales", "sociedad de la información", "sociedad del conocimiento", "formación docente", "nuevos paradigmas de formación docente", "la socioformación docente", "educación sostenible", "sostenibilidad", "complejidad social", "comunidad", "comunidad de aprendizaje", "dialogicidad", "espacios dialógicos", etc.

La selección fue basada en artículos de revistas indexadas que abordan temas referentes a nuestras categorías de investigación y análisis, producidas en los últimos cinco años. Durante la búsqueda y selección de la información recabada, fue necesario hacer un análisis de los subtemas o subcategorías relacionados con las categorías principales de nuestra investigación, como fue el caso de "los problemas del contexto", "medio ambiente", "economía social", "capitalismo", "consumismo", entre otros.

Los documentos utilizados en nuestro análisis documental, se describe a continuación como una síntesis cuantitativa para el estudio realizado, se consideran aquellos documentos que cumplieron con los criterios científicos de investigación y fue necesario revisar artículos en su mayoría de la ubicación contextual geográfica donde se lleva a cabo la investigación, sin dejar de lado, aunque en menor cantidad artículos de otras regiones, los cuales se exponen a continuación. (ver Tabla 2). 
TABLA 2. Ejemplo de Documentos Analizados en el Estudio

\begin{tabular}{|l|c|c|c|c|}
\hline \multicolumn{1}{|c|}{ DOCUMENTOS } & SOBRE EL TEMA & $\begin{array}{c}\text { DE } \\
\text { CONTEXTUALIZACIÓN } \\
\text { O COMPLEMENTO }\end{array}$ & LATINOAMERICANOS & DE OTRAS REGIONES \\
\hline Artículos teóricos & 18 & 4 & 4 & 15 \\
\hline Artículos empíricos & 38 & 4 & 3 & 12 \\
\hline Libros & 1 & 1 & 2 & 2 \\
\hline Manuales & 1 & 1 & 1 & 0 \\
\hline
\end{tabular}

Elaborado por los autores para la presente investigación

\section{DESARROLLO}

\section{Socioformación}

La palabra socioformación se compone de los vocablos "Social" y "Formar", considerando su etimología, "Social" del lat. sociětas, -ātis agrupación natural o pactada de personas, organizada para cooperar en la consecución de determinados fines, y "formar" del lat. formāre. dicho de una persona, adquirir preparación intelectual, moral o profesional (RAE, 2018). Bajo esta premisa, se plantea una nueva perspectiva latinoamericana, y que considera el desarrollo del talento humano que contribuya a la formar personas para vivir e interactuar en una sociedad del conocimiento "para afrontar los retos del contexto local global, con el fin de lograr la buscando la calidad de vida, el tejido social la convivencia, la inclusión y el desarrollo socioeconómico, teniendo como base en el aseguramiento de la y el equilibrio y sustentabilidad ambiental-ecológica."(Salazar-Gómez y Tobón, 2018).

Tobón $(2015$, p.12), define la socioformación como la unión de ambos términos "enfatizando en el desarrollo de las personas en un contexto social, pero un contexto social que no es el aula o la escuela; es el contexto de la sociedad real, con sus problemas". Es por esto que desde este nuevo enfoque se busca que los individuos desarrollen sus talentos, capacidades y potencialidades, y se realicen plenamente resolviendo problemas de la sociedad real, con base en sus propias vivencias, seleccionando áreas concretas de actuación. En estas áreas concretas de actuación, se destaca el contexto con el que todo individuo interactúa y que supone una formación integral del mismo donde se destaca particularmente el abordaje de los problemas del contexto buscando fortalecer el trabajo colaborativo como eje de un trabajo transversal (Tobón, González, Nambo, Vázquez, 2015).

Es importante señalar algunas características que definen o identifican a la Socioformación. Tobón (2013c, 2013d) define cinco características claves sobre el enfoque socioformativo. 1) Proyecto ético de vida, emprendimiento, gestión y co-creación del conocimiento, trabajo colaborativo y metacognición. El Proyecto ético de vida (Lomeli Parga, Lopez, \& Valenzuela, 2016) sólido es entendido como la forma de vivir buscando la plena realización personal y contribuir al tejido social, la paz y el desarrollo socioeconómico, buscando el equilibrio y sustentabilidad ambiental. Implica actuar con los valores universales, desde la perspectiva ética defiende entonces la actuación de la moral y el comportamiento humano rescatando el pensamiento crítico y el humanismo.

2) El emprendimiento (Salazar, González, Sánchez, \& Sanmartin, 2018) es definido como el proceso por medio del cual se inician y sacan adelante proyectos que contribuyan a resolver problemas del contexto, con creatividad, pertinencia, eficiencia y eficacia. También es necesario rescatar la relevancia y viabilidad para poder llevarlos a cabo sin perder de vista que estos deberán contribuir en la mejora y cuidado del medio ambiente. Su desarrollo requerirá entonces del 3) trabajo colaborativo (Vázquez, Silvano, Vázquez, Juárez, \& Guzman, 2016), pues es necesario que estos proyectos surjan de las propias necesidades del grupo o comunidad en cuestión, para lo cual será necesario establecer planes de acciones con actividades que articuladas que involucren de manera responsable y comprometida a los miembros del mismo para el logro de metas u objetivos comunes.

Al momento de llevar a cabo el desarrollo de proyectos o planes de trabajo, es necesario rescatar la 4) gestión y co-creación del conocimiento, el cual Tobón $(2015$, p.16) lo define como "aquel proceso por medio del cual se busca, procesa, guarda, comprende, adapta, crea, innova y aplica el conocimiento de diversas áreas en la resolución de problemas del contexto con análisis sistémico, ética y colaboración". Por tanto, visto de esta manera se somete a la conjunción y aprovechamiento de los saberes que los miembros del grupo o comunidad tienen y ponen de manifiesto al momento de analizar y aplicar sus conocimientos, aprovechando de esta manera uno de los ejes fundamentales de la sociedad del conocimiento (Sterch, 1994), la búsqueda, organización y construcción del saber.

Otra característica es la 5) Metacognición (Tobón, 2013, p.15), la cual es entendida como "el proceso por medio del cual las personas mejoran continuamente su desempeño para alcanzar unas metas por medio de la reflexión y la guía de unos determinados valores o referentes externos". De esta manera se genera nuevos conoci- 
mientos sometidos a análisis y resultados, los cuales se superan a sí mismos, pues implican la toma de consciencia o autorregulación, porque involucra acciones reales de mejora con base en metas comunes, trabajo colaborativo e interdependencia.

\section{Formación docente}

La formación docente presenta nuevos desafíos debido a que estos tienen que estar preparados para enfrentar los retos de las sociedades del conocimiento, donde el fin de la formación radica en formar profesionales con altas expectativas de formación académica con las competencias profesionales para desenvolverse en su práctica docente. Esto plantea la necesidad de la preparación docente, lo que implica el desarrollo de competencias profesionales para desarrollar sus prácticas docentes orientados en la formación de ciudadanos rescatando la ética, y los valores. (Salazar-Gómez \& Tobón, 2018).

El docente desempeña un papel importante porque se requiere de ser facilitador en los procesos de enseñanza y aprendizaje, debe de estar en constante evolución y cambio, ofreciendo herramientas para el aprendizaje con nuevas formas de aprender. El docente e un sujeto transformador de la realidad, por lo tanto, se espera que estén comprometidos con su trabajo. Es el responsable de orientar su trabajo en el desarrollo humano para trascender y el desarrollo de competencias para estar preparados para los desafíos que se presentan en la sociedad del conocimiento. (Nieva Chaves, J. A., \& Martínez Chacón, 2016).

La profesionalización del docente (Morales, 2018), es una movilización de saberes y actitudes para utilizar una destreza que serán utilizadas en su labor educativa. Es un aprendizaje continuo y dinámico, o con ciertas competencias para el desempeño de su función. La profesionalización docente refiere a un concepto positivista de una práctica que utiliza la investigación científica y la aplicación de teorías a la resolución de problemas (García-Esteban \& Murga-Menoyo, 2015). La formación docente en la sostenibilidad pretende que tengan las características necesarias para los retos que se enfrentan ante la resolución de problemas de tipo ambiental, social, económico, educativo, desigualdad social, mediante un pensamiento más complejo y sistémico. (Tobón, 2017).

Para Sánchez (2013, p.21), la formación inicial y la formación continua son dos categorías fundamentales en la formación y el desarrollo profesional docente; "la formación inicial es la que se encarga de preparar profesionalmente a los estudiantes para la docencia escolar, insertándose en el ámbito pedagógico a través de programas específicos que habilitan y certifican su condición de docentes ante la sociedad". Constituye el comienzo de la trayectoria formativa profesional docente, es por medio de ésta, que el futuro maestro acoge las herramientas teórico-metodológicas para desarrollar su práctica en un campo pedagógico específico a través de programas educativos formales. Sin embargo, el currículo actual dista de dotar a los docentes de una formación con un sentido sostenible para enfrentar los retos de las sociedades actuales, desarrollar en ellos el uso del pensamiento crítico para atender problemas socioambientales cada vez más complejos (Alberto, 2016).

La formación continua, dispone de dotar a los docentes del desarrollo de sus necesidades identificadas en su trayecto laboral, de cara a su propia profesionalización; sin embargo, ésta se orienta en la atención de problemas de carácter procedimental de la didáctica y su intervención docente. Dos Santos, Schlünze, \& Junior (2016, p. 34), señalan que "esta se puede entender como la acción de aprendizaje posterior a la educación inicial, en el cual el docente puede recibir actualización y elementos para desarrollar su práctica profesional; en esta etapa se encuentran los posgrados, las especializaciones y otras actividades de índole formativa como las conferencias y asistencia a cursos relacionados con la docencia". De esta manera y acorde a la sociedad del conocimiento, ambas acciones de formación docentes deberán desde la etapa inicial, como en la propia formación continua, permitir transformarse, re-pensarse y conducirse en la atención de los retos sociales, económicos, ecológicos, políticos, culturales y tecnológicos.

De manera tradicional el rol que el docente siempre ha desempeñado, ha sido como el de la única fuente proveedora del conocimiento en el aula, el experto cuya experiencia es inobjetable; en la actualidad esta postura no es la más adecuada pues las mismas exigencias y cambios en los paradigmas sociales, aunados al avance científico y tecnológico requiere de un docente cuyo papel o rol se transforme en un facilitador del aprendizaje de sus alumnos, que los motive para trabajar de manera colaborativa, fomentando en ellos la apreciación de sus propias fortalezas para su aprendizaje y sobre todo para la resolución de problemas concretos. En este sentido Parra, Acosta, Tobón, López \& Loya (2016), señalan que "el profesor, más que un instructor, es un mediador generador de ambientes de formación flexibles, dinámicos y estimulantes. Así, se busca que los alumnos desarrollen competencias sustentables, que hacen referencia a la actuación integral para resolver situaciones en situaciones complejas y cambiantes".

\section{Desarrollo sostenible}

El desarrollo sostenible es concepto básico que surge para la sociedad para tratar de resolver los problemas globales que se presentan como lo son el cambio climático, la economía, lo social como la pobreza, la desigualdad, la educación, para garantizar una esperanza de vida para las próximas generaciones. Este es un proyecto pretende comprender las interacciones desde tres sistemas que son: la economía, la sociedad y el ambiental, con un enfoque holístico (Daza, 2016), porque promueve los valores de preservación de la biodiversidad, la diversidad humana, la inclusión, la participación de los miembros de la sociedad para reducir las desigualdades en los seres humanos 
(Sachez, 2015).

La educación para el desarrollo sostenible permite que las personas adquieran conocimientos, habilidades, competencias para un futuro sostenible, es decir que se incorpore en la enseñanza temas de impacto social para combatir el cambio climático, la pobreza, el consumo sostenible, reducir las desigualdades, fomentar el pensamiento crítico y complejo para que los alumnos modifiquen esquemas tradicionales y formas de vida que sean colaborativos como: la conservación, la regeneración y la resiliencia para la preservación de la vida humana implementando un sistema de educación que permita la sostenibilidad en la educación (Sachez, 2015).

Esta propuesta del desarrollo sostenible se caracteriza por ser sistémica y global, porque está constituida por principios éticos, para la preservación de la biodiversidad ambiental, así como la promoción de la equidad social y los derechos humanos basados en una democracia de las personas que conforman la sociedad para una mejora de vida y de las generaciones futuras, (Mínguet \& Ruiz, 2017). Este planteamiento está fundamentado en un crecimiento tanto social como económico para promover el bienestar común y ampliar las oportunidades de las personas para una mejor calidad de vida (Sanches, 2015).

Los criterios referentes a la capacitación para la sostenibilidad son: comprender que lo primordial es el cuidado de la sociedad y del medio ambiente, identificando los riesgos, desafíos e impactos. El entender la contribución de su trabajo en los diferentes ámbitos poniendo la ética del cuidado para la preservación de la vida humana. Otro de los criterios es trabajar en equipos multidisciplinares para dar solución a los problemas socioambientales, estos derivados de los estilos de vida del ser humano. (Muñoz, Callejo, Sastre, \& Marín, 2017).

En la actualidad la sostenibilidad del sistema humano, transita en la gama de recursos y servicios que pueden sustituirse íntegramente, desde este punto de vista que "la tierra pudiera convertirse en un planeta totalmente artificial" (Gallopin, 2003). Desde un enfoque económico, el sistema que gobierna es el económico, y desde este punto de vista, la naturaleza "se relega a la función de proveedora de recursos y servicios naturales y a sumidero de los desechos producidos por la actividad humana" (Turner, 1993). De esta manera, el reto que enfrenta hoy las sociedades humanas será replantar el capital natural por encima de capital manufacturado

\section{Comunidades de aprendizaje}

En la actualidad, las comunidades de aprendizaje se definen como "una respuesta eficiente y equitativa a los cambios y retos sociales y educativos introducidos por la sociedad de la Información" (Díez-Palomar \& Flecha García, 2010), diferente de las aulas de la sociedad industrial, en las aulas y otros espacios de las comunidades de aprendizaje existen personas adultas comprometidas con la educación de todos los niños. La interactividad generada en un grupo, es un ejemplo de ello. En estos grupos pequeños y heterogéneos los estudiantes colaboran de forma dialógica para resolver actividades de aprendizaje con la ayuda de personas adultas (Aubert y García, 2001)

El diálogo con los docentes a través de círculos de estudio, reuniones colegiadas, etc. pone en juego un sin número de conocimientos y experiencias que cada uno de ellos tiene, esta práctica permite transformaciones, pero no hay que olvidar que muchas veces no se incluye a todos en la toma de decisiones o de cambios educativos o del entorno que los afectan. Señala que muchas instituciones se han burocratizado y que nacieron fruto de las reivindicaciones de la población y el diálogo, una vez institucionalizadas han puesto barreras a la población de la cual surgieron para intervenir (Pérez, 2010).

\section{CONCLUSIONES}

A partir del análisis documental llevado a cabo, una primera conclusión es que es necesario entender la socioformación como un enfoque eminentemente social y colaborativo (Prado, 2018), donde la tarea principal es afrontar los retos cada vez más complejos de nuestra sociedad. A partir de este es necesario proponer y ejecutar espacios de dialogo profesional en vistas de alcanzar una sociedad mas sostenible (Riechmann, 2019).

Una segunda conclusión es que, en la actualidad, la preparación académica de los docentes, así como su formación continua, no permite el desarrollo de un pensamiento crítico (Moreno-Pinado \& Velázquez Tejeda, 2017) que trascienda más allá de la enseñanza de contenidos; aunado a la vision referida por las políticas educativas actuales, centradas más en la observancia de resultados de índole cuantitativos (Ávila-Gómez, 2016), en resultados educativos orientados por indicadores como deserción, reprobación, rezago que sin duda son factores que se gestan en un ámbito eminentemente social y que se convierten en problemáticas más humanistas (Ortiz Bosch, López Rodríguez, \& Álvarez Cortés, 2016) que cognoscitivas.

En tercer lugar, puede considerar la necesidad urgente de revisar y atender el currículo actual de la formación y profesionalización docente, desde su fase inicial hasta la formación continua, pues se requiere hacer aliado a un sistema educativo sostenible (Guillen, 2004), enfocado en desde la escuela fomentar el desarrollo de ciudadanos más críticos y reflexivos (Collado Ruano, 2016), capaces de ser conscientes y atender los problemas de su entorno 
para garantizar la preservación de la humanidad. Replantear el currículo educativo supone la necesidad de transitar de una sociedad de la información a una sociedad del conocimiento, pero también una sociedad del riesgo.

Al realizar nuestro análisis documental, nos percatamos que existe poca información en investigaciones hechas respecto a la formación docente desde un enfoque socioformativo y a la creación o desarrollo de comunidades de aprendizaje dialógicas con este mismo enfoque, pero que además sean capaces de integrar a la comunidad (Vieira Parra \& Puigdellívol, 2013); por lo que es necesario proponer nuevos estudios en el área, rescatando principalmente el énfasis en la sostenibilidad la educación, es decir un sistema educativo capaz de atender no solamente contenidos disciplinares, si no dotado de pensadores humanos (Sierra Villamil, 2016), conscientes de entender los avances científicos y tecnológicos asumiendo los riesgos y desafíos presentes para sus atención.

\section{REFERENCIAS BIBLIOGRÁFICAS}

Ávila-Gómez, M. (2016). La evaluación de calidad como medio de transformación educativa. Revista Educación, Política y Sociedad.

Carmen, E. S., \& Perez, E. O. (2003). Comunidades de aprendizaje un modelo de educación dialógica en la sociedad del conocimiento. Estados Unidos.

CNSPD. (Marzo de 2019). Servicio Profesional Docente. Obtenido de http://file-system.cnspd.mx/2019-2020/ingreso/ba/misc/PPI_ EB_2019_20191202.pdf

Coll, C. (Abril de 2010). Enseñar y aprender en el mundo actual: desafios y encrucijadas. Pensamiento Iberoamericano. Obtenido de https://s3.amazonaws.com/academia.edu.documents/31952726/CC_2010_Pensamientolberoamericano.pdf?AWSAccessKeyld=AKIAIWOWYYGZ2Y53UL3A\&Expires=1552015350\&Signature=YmM7v5pMgO6dYVzL58DglqoApYw\%3D\&response-content-disposition=inline\%3B\%20filename\%3DCC_2010_Pens

Collado Ruano, J. (2016). Epistemología del Sur: una visión descolonial a los objetivos de desarrollo sostenible. Revista de História da África e de Estudos da Diáspora African, 1-26.

Delors, J. (1993). La educación encierra un tesoro. Santillana.

DGESPE. (2018). Recuperado el 19 de 03 de 2019, de Dirección General de Educación Superior para Profesionales de la Educación: https://www.cevie-dgespe.com/index.php/planes-de-estudios-2018

Díez-Palomar, J., \& Flecha García, R. (2010). Comunidades de Aprendizaje: un proyecto de transformación social y educativa. Revista Interuniversitaria de Formación del Profesorado, 19-30.

Díez-Palomar, J., \& Flecha García, R. (2010). Comunidades de Aprendizaje: un proyecto de transformación social y educativa. Revista Interuniversitaria de Formación del Profesorado, 19-30.

Díez-Palomar, J., \& Flecha García, R. (2016). Comunidades de Aprendizaje: un proyecto de transformación social y educativa. Revista Interuniversitaria de Formación del Profesorado, 12.

Dulzaides, M. E., \& Molina, A. M. (01 de 04 de 2019). Análisis documental y de información: dos componentes de un mismo proceso. Obtenido de Scielo: http://scielo.sld.cu/pdf/aci/v12n2/aci11204.pdf

España, E., \& Prieto, T. (2009). EDUCAR PARA LA SOSTENIBILIDAD: EL CONTEXTO DE LOS PROBLEMAS SOCIO-CIENTÍFICOS. Eureka, 345-354.

Esteve, J. M. (2015). La profesión docente ante los desafios de la sociedad del conocimiento. Aprendizaje y desarrollo profesional docente. Madrid, España: Fundación Santillana.

Gallopin, G. (2003). Sostenibilidad y desarrollo sostenible: Un enfoque sistémico. Santiago de Chile: CEPAL.

Gallopín, G. (2003). Sostenibilidad y desarrollo Sostenible: un enfoque sistémico. Santiago de Chile: ONU.

Gudynas, E. (2003). Ecología, economía y etica del desarrollo sostenible. Quito Ecuador: ILDIS-FES.

Guillen, F. C. (2004). Educación, medio ambiente y desarrollo sostenible. Revista Iberoamericana de Educación, 1-7.

Hernandez, J. S., Tobón, S., \& Vazquez, J. M. (2014). Estudio conceptual de la docencia socioformativa. 10(5). (R. Ximhai, Ed.) México.

Hernández-Mosqueda, J. S., Tobón-Tobón, S., \& Vázquez-Antonio, J. M. (2014). Estudio conceptual de la docencia socioformativa. El Fuerte, México.

Krüger, K. (2015). El concepto de sociedad del conocimiento. Barcelona, España. Obtenido de https://www.researchgate.net/publication/245535884

Lomeli Parga, A., Lopez, M., \& Valenzuela, J. (2016). Autoestima, motivación e inteligencia emocional: Tres factores influyentes en el diseño exitoso de un proyecto de vida de jóvenes estudiantes de educación media. Revista Electrónica Educare.

Luna_Nemecio, J. (2019). Calentamiento Global y conflictividad sociohídrica ante el colapso ambiental del estado de Morelos, México. Revista Latinoamericana de Estudiantes de Geografía, 74-84.

Moreno-Pinado, W. E., \& Velázquez Tejeda, M. E. (2017). Estrategia Didáctica para Desarrollar el Pensamiento Crítico. Revista Iberoamericana sobre Calidad, Eficacia y Cambio en Educación, 53-73.

Olivé, L., \& Ransanz, A. R. (2011). Las sociedades humanas en el siglo XXI: Complejidad, incertidumbre y desafíos. Artículo. México.

Ortiz Bosch, M. J., López Rodríguez, J., \& Álvarez Cortés, A. B. (2016). LA FORMACIÓN HUMANISTA DE LOS ESTUDIANTES DE CIENCIAS PEDAGÓGICAS. Didáctica y Educación.

Pedraja Rejas, L. (Abril de 2012). Desafíos para el profesorado en la sociedad del conocimiento. 20(1). (R. C. Ingeniería, Ed.) Arica, Chile.

Pedraja Rejas, L. (Abril de 2012). Desafíos para el profesorado en la sociedad del conocimiento. Arica, Chile. Obtenido de http:// www.redalyc.org/articulo.oa?id=77222768014

Perez, E. O. (2010). Las comunidades de aprendizaje, un modelo de educación dialógica de la sociedad del conocimiento. 
Perrenoud, P. (2004). Diez nuevas competencias para enseñar. Queretaro, México.

Prado, R. A. (2018). La socioformación: un enfoque de cambio educativo. Revista iberoamericana de educacion, 57-82.

Racionero, S., \& Serradell, O. (2005). Antecedentes de las comunidades de aprendizaje. Educar, 35-39.

RAE. (2018). Diccionario de la lengua española. Recuperado el 19 de Marzo de 2019, de https://dle.rae.es

RAE. (19 de 03 de 2019). Diccionario de la lengua español. Obtenido de https://dle.rae.es/?id=AVBbFZW

Riechmann, J. (06 de Marzo de 2019). Desarrollo Sostenible: La Lucha. Obtenido de http://www.istas.ccoo.es/descargas/desost.pdf

Salazar, F., González, J., Sánchez, P., \& Sanmartin, J. (Diciembre de 2018). Emprendimiento e Innovación: Agentes potenciadores de la empresa familiar.

SEP. (2011). Plan de Estudios 2011. 36. México.

Servaes, J. (Abril de 2012). Comunicación para el desarrollo sostenible y el cambio social: una visión general. Madrid, España: Universidad Complutense de Madrid.

Sierra Villamil, G. M. (2016). Liderazgo educativo en el siglo XXI, desde la perspectiva del emprendimiento sostenible. Artículos científicos, 1-18.

Tobón, S. (2010). Proyectos formativos: Metodología para el desarrollo y evaluación de competencias. México.

Tobón, S. (Junio de 2015). La socioformación: análisis documental de su impacto en lberoamérica. Chihuahua, México.

Tobón, S. (2015). La Socioformación: un estudio conceptual. Cuernavaca, Morelos, México: Centro Universitario CIFE.

Tobón, S., Gonzalez, L., Nambo, J. S., \& Antonio, J. M. (2015). La Socioformación: Un Estudio Conceptual. Paradigma, XXXVI(1).

UNESCO. (2005). Hacia las sociedades del conocimiento. 17-24.

Vázquez, J. M., Silvano, H. J., Vázquez, J., Juárez, L. G., \& Guzman, C. E. (2016). El trabajo colaborativo y la socioformación: un camino hacia el conocimiento complejo. Revista Educación y Humanismo.

Vieira Parra, L., \& Puigdellívol, I. (2013). ¿Voluntarios dentro del aula? El rol del voluntariado en "Comunidades de Aprendizaje". 1-19.

Villavicencio, D., Morales, A., \& Amaro, M. (2012). Indicadores y asimetrías sobre la Sociedad Basada en el Conocimiento en América Latina. Perfiles latinoamericanos, 63-95

Wallerstein, I. (1988). El capitalismo histórico. Madrid: Siglo XXI de España Editores, S. A.

How to cite this article: Vazquez-Ayala, D. (2020). La Formación Docente Socioformativa para el Desarrollo Social Sostenible. Religación. Revista De Ciencias Sociales Y Humanidades, 5(23), 96-104. Recuperado a partir de http://revista.religacion. com/index.php/religacion/article/view/602

Submitted: 07 January 2020 Accepted: 14 March 2020 Published: 31 March 2020

Religación. Revistais de Ciencias Sociales y Humanidades is a peer-reviewed open access journal published by CICSH-AL Centro de Investigaciones en Ciencias Sociales y Humanidades desde América Latina |Religación| 